\title{
Saccade Preparation Inhibits Reorienting to Recently Attended Locations
}

\author{
Robert D. Rafal, Peter A. Calabresi, Cameron W. Brennan, and Toni K. Sciolto \\ Department of Clinical Neurosciences, Roger Williams General Hospital \\ Providence, Rhode Island, and Brown University Program in Medicine
}

\begin{abstract}
We measured manual reaction time in normal human subjects to confirm that an eccentric visual signal has a biphasic effect on covert attention and eye movements. First, it summons attention and biases a saccade toward the signal; a subsequent inhibition of return then slows responses to signals at that location. A temporal hemifield dominance for inhibition of return was shown; this finding coverges with observations in neurologic patients to suggest that it is mediated by midbrain pathways. Endogenous orienting of attention, from a central arrow cue, did not activate inhibition of return, whereas endogenous saccade preparation did so as effectively as an exogenous signal, even when no saccade was made. Inhibition of return is activated by midbrain oculomotor pathways and may function as a location "tagging" mechanism to optimize efficiency of visual search.
\end{abstract}

The selectivity of visual perception is guided by brain mechanisms that orient attention in the visual field. One manifestation of visual orienting is overt, consisting of movements of the head or eyes, or both, to focus on the attended spatial position. Covert orienting may occur, however, independently of motor activity and serves to align attention to a spatial location to enhance the processing of or response to signals that occur there. Researchers have studied covert orienting in humans by measuring the pattern of facilitation and inhibition in responding to an eccentric visual target, which result from preliminary cues that either correctly prepare the subject to detect the target at the cued location or summon attention elsewhere in the visual field (Posner, 1980). In this kind of experiment, the eyes remain fixed in the center of the display, and inasmuch the required response is always a simple reaction time (RT) manual key press regardless of target location, these facilitations and inhibitions in RT performance serve as a measure of visual attention that is independent of eye position or response selection. This method has also been used in experiments in which eye movements are made to show that when a peripheral signal is the target of a saccade, attention moves to the signal before the onset of the saccade (Posner \& Cohen, 1980) and that under some circumstances, attention can move in one direction while the eyes move in another (Posner, 1980).

A major goal of research in visual attention, addressed in this study, is to determine how neural systems responsible for the covert allocation of visual attention are integrated with

This work was supported by Public Health Service Grant R01 MH 41544 and by Roger Williams General Hospital. We are grateful to Michael Posner for his guidance throughout the conduct of this work. Corrine Hopp assisted in preparation of the manuscript of this article.

We are grateful to Ray Klein and James Cutting for their suggestions for control experiments and for their considerable effort in helping to improve this article.

Correspondence concerning this article should be addressed to Robert D. Rafal, Neurology Department, Martinez Veterans Administration Hospital, 150 Muir Road, Martinez, California 94553. oculomotor systems for overt orienting behavior. It has been shown that an eccentric visual signal has a biphasic effect both on the covert orienting of attention and on eye movements. First, the cue summons attention automatically to facilitate detection at the locus of the signal (Posner, Cohen, \& Rafal, 1982) and biases midbrain oculomotor centers to prepare a saccade toward it (Posner \& Cohen, 1980). These facilitory effects are followed by an inhibition that slows detection at the cued location and induces a bias against making a saccade toward it (Maylor, 1985; Maylor \& Hockey, 1985; Posner \& Cohen, 1984; Posner, Rafal, Choate, \& Vaughan, 1985; Tassinari, Aglioti, Chelazzi, Marzi, \& Berlucchi, 1987).

Posner and Cohen (1984) called this later inhibitory effect inhibition of return and suggested that it modulates oculomotor activity by favoring novelty in visual scanning. They showed that the effect lasted at least $1,500-2,000 \mathrm{~ms}$, long enough to influence several saccades. It influences saccade latency (Vaughn, 1984) and direction (Posner et al., 1985), but not perceptual (temporal order) judgments (Posner et al., 1985). Peritectal degeneration in neurologic patients with progressive supranuclear palsy produced a deficit in the inhibition of return in the same directions in which eye movements were most severely impaired, whereas inhibition of return was not disrupted in control patients with Parkinson's disease or with lesions of the frontal, temporal, or parietal lobes (Posner et al., 1985).

Although the inhibition of return appears to be linked to covert shifts of attention summoned by an eccentric visual signal, it does not necessarily occur with all movements of visual attention. Specifically, it does not occur with endogenously activated shifts in attention in response to symbolic cues (an arrow in the center of the display that instructs the subject where to expect a forthcoming target; Posner \& Cohen, 1984). However, if an arrow cue induces the subject to make a saccade to an eccentric location and the eyes are then returned back to the initial fixation point, inhibition of return does occur for subsequent detection at the location to which the saccade had been made (Posner et al., 1985). Thus inhi- 
bition of return may be activated either by an exogenous sensory signal presented in the visual periphery while the eyes are fixed or by the endogenous activation of a saccadic eye movement.

In this investigation we sought to clarify the relation between eye movement systems and the inhibition-of-return phenomenon and to test the hypothesis that it serves as a mechanism for integrating visual attention and eye movements. Because we have shown previously, in neurologic patients, that lesions of midbrain oculomotor centers prevent generation of inhibition of return (Posner et al., 1985), we first sought converging evidence that these centers may mediate the inhibition of return in normal humans. In our first three experiments, we showed a temporal hemifield dominance for inhibition of return, which is consistent with known connections of midbrain visual pathways. In subsequent experiments, we identified a common mechanism mediating inhibition of return by exogenous or endogenous activation, namely, priming the oculomotor system to prepare a saccade.

\section{Experiment 1: Inhibition of Return in Nasal and Temporal Hemifields}

We performed Experiment 1 to confirm that the inhibition of return is mediated through the extrageniculate visual system by demonstrating a greater effect in the temporal hemifield under monocular conditions. In contrast to the equally binocular geniculostriate system, the extrageniculate retinotectal pathway is essentially monocular; each colliculus receives dominantly crossed input from the contralateral retina. In monkeys the temporal hemiretina (nasal hemifield) has a smaller direct input to the superior colliculus (Hendrickson, Wilson, \& Toyne, 1970; Hubel, LeVay, \& Wiesel, 1975; Pollack \& Hickey, 1979; Wilson \& Toyne, 1970). There is evidence for such a monocular temporal hemifield dominance in the human retinotectal pathway in both newborn infants (Lewis, Maurer, \& Milewski, 1979) and normal adults (Posner \& Cohen, 1980). If, as our results in neurological patients suggest, the inhibition of return is mediated by the extrageniculate visual system of the midbrain, a temporal bias for the effect under monocular conditions would be expected in normal humans.

\section{Method}

Subjects. Twenty normal, young adult volunteers were paid to participate in this experiment under monocular conditions. Subjects were recruited from among employees, students, and staff of the Roger Williams General Hospital.

Apparatus. Subjects sat in a dark room facing a black and white video monitor $36 \mathrm{~cm}$ in front of them at eye level, which was interfaced with an Apple II microcomputer that generated stimuli on the monitor and recorded RT responses on-line. The head rested loosely on a chin rest, and the index finger of the preferred hand rested on a response key on the table between the subject and the display.

Procedure. Each eye was tested in separate blocks of 240 trials, and the order of eye patching was randomized across subjects. The display consisted of a $1^{\circ}$ unfilled square box at central fixation flanked, $10^{\circ}$ to left and right, by two other $1^{\circ}$ square boxes. Subjects were

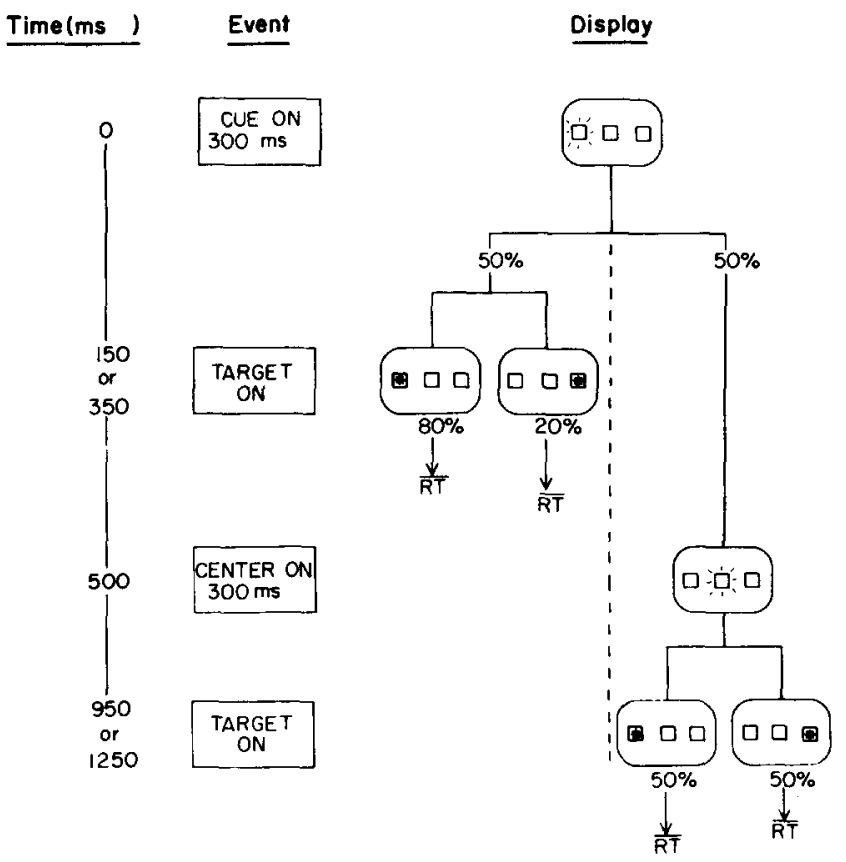

Figure 1. Design of Experiment 1 (see text).

instructed to maintain eye fixation on the center box and to respond with a simple RT key press to the appearance of a large, bright target (an asterisk filling the box) that appeared, with equal probability on each trial, in either of the two flanking boxes. Eye position was monitored by video camera to ensure that subjects did not move their eyes.

In Figure 1 we show the display used and the design of the experiment. After an intertrial interval of $3,000 \mathrm{~ms}$, each trial began with the brightening of one of the two peripheral boxes for $300 \mathrm{~ms}$. This signal cued the subject to prepare to detect a target at that location. In half of the trials, the target appeared after this first cue (either 150 or $350 \mathrm{~ms}$ after cue onset) and was plotted, $80 \%$ of the time, at the cued location, whereas the other $20 \%$ of the time, it occurred in the uncued contralateral box.

However, in the other half of trials, the target did not appear after the first cue. Instead, the center box brightened $500 \mathrm{~ms}$ after onset of the first cue for $300 \mathrm{~ms}$. The purpose of the second cue was to summon attention back to the center. The central brightening was then followed by the target (either 950 or $1,250 \mathrm{~ms}$ after onset of the first cue). In these center-brightening (double-cue) trials, the target appeared with equal probability either at the first cued location or in the contralateral box. On every trial the target remained visible until the subject responded. There were no catch trials.

In this experiment, then, there were two types of trials: (a) In the single-cue trials, the target followed a peripheral cue and was more likely $(80 \%)$ to appear at the cued location than in the contralateral box. This probability manipulation was contrived to induce subjects to expect the target at the cued location and to move their attention to it, even though their eyes stayed fixed at the center. On these single-cue trials, the effectiveness of the peripheral cue in summoning attention was inferred from relatively faster detection RTs at the cued location. (b) In the double-cue trials, attention was summoned back to the center by the brightening of the center box, and the target was then equally likely to appear at either peripheral location. On these center-brightening trials, inhibition of return was inferred from the 
relatively slower detection $\mathrm{RT}$ s at the first cued location from which the subject had just withdrawn attention.

\section{Results}

RTs of less than $100 \mathrm{~ms}$, assumed to be anticipatory responses, or greater than $3,500 \mathrm{~ms}$ were excluded from subsequent analysis. These trials accounted for less than $4 \%$ of the data. Then the mean reaction times for each subject, for each condition of cue and interval, were subjected to a $2 \times 2 \times 4$ repeated-measures analysis of variance (ANOVA). Within-subject factors included hemifield (temporal or nasal), cue validity (target appearing at the location of the first cue or cue appearing contralateral to the cued location), and interval $(150,350,950$, or $1,250 \mathrm{~ms})$.

In Figure 2 we show the mean results for all 20 subjects. There was no main effect of hemifield. Reaction times were faster at the later cue-target intervals, $F(3,57)=17.0, p<$ .001 . Cue interacted with interval, $F(3,57)=20.0, p<.001$, in such a way that reaction times were quicker at the cued location for the two intervals that followed the first cue, and slower at the cued location for the two intervals that followed the center's brightening. This result confirms that the cue was effective in summoning attention initially and in activating an inhibition of return. The data from the two cue-target intervals that followed the first cue and those from the later two intervals that followed the center's brightening were cast into separate ANOVAs. Within each of these two types of trials,

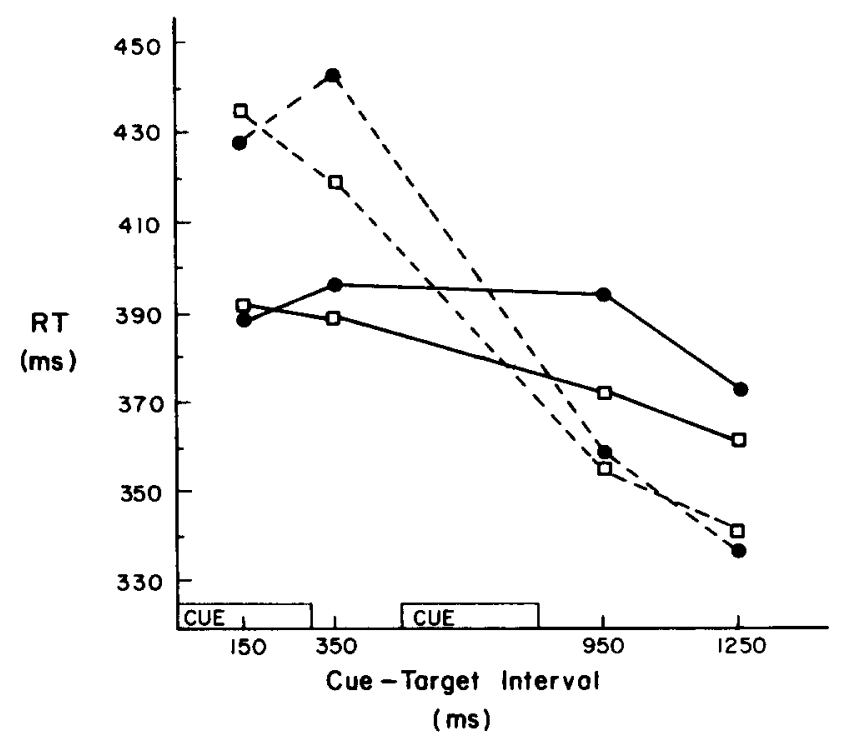

Figure 2. Mean reaction times (RTs) from Experiment 1. (RTs for targets appearing at the location of the cue [solid lines] and contralateral to the cue [dashed lines] in the temporal hemifield [closed circles] and the nasal hemifield [open squares] are shown as a function of cue-target interval [stimulus onset asynchrony in ms]. The timing of the first [peripheral] cue and the second [center-brightening] cue are shown. The quicker responses to targets at the location of the cue for the single-cue trials show the initial orienting of attention; the slower responses to targets at the location of the cue for the doublecue trials show the inhibition of return.) there were no main effects of cue-target interval or interactions with interval.

Single-cue trials. For the trials that followed the first cue, RTs were $\mathbf{4 0}$ ms quicker for targets at the location of the first cue than in the contralateral field, $F(1,19)=34.4, p<.001$. There were no significant interactions between cue effect and hemifield for these single-cue trials.

Center-brightening trials. For the double-cue trials, in which targets followed a center's brightening, RTs were slower at the location of the first cue, $F(1,19)=34.4, p<.001$. This inhibition-of-return effect (RT to targets appearing at the location of the first cue minus RT to targets in the field contralateral to the cue) interacted with hemifield, being larger in the temporal hemifield ( $36 \mathrm{~ms}$ ) than in the nasal hemifield $(20 \mathrm{~ms}), F(1,19)=5.0, p<.05$. Separate ANOVAs done on the data for the temporal hemifield and nasal hemifield targets on the center-brightening trials confirmed that the inhibitionof-return effect was significant within each field: $F(1,19)=$ $24.3, p<.001$, for the temporal hemifield, and $F(1,10)=$ 22.2, $p<.001$, for the nasal hemifield. According to Figure 2 , for targets appearing at the uncued location, there is little difference between temporal and nasal hemifields, and the larger inhibition of return effect in the temporal hemifield (derived from the paired comparison) appears to be due to slower RTs to targets at the cued location in the temporal hemifield than in the nasal hemifield. However, a separate ANOVA done on data from trials with targets appearing at the cued location revealed that this difference between the two hemifields did not achieve statistical significance $(F=1.8$, $n s)$.

\section{Discussion}

The results of Experiment 1 confirm the previously reported observations that a peripheral visual signal first summons attention and then results in an inhibition of responses to subsequent signals at the cued location (Maylor, 1985; Maylor \& Hockey, 1985; Posner \& Cohen, 1984; Posner et al., 1985; Tassinari et al., 1987). The purpose of Experiment 1 was to determine whether there were differences between the temporal and nasal hemifields, either for the initial orienting of attention (on the single-cue trials) or for the subsequent inhibition of return (on the double-cue trials). In a previous study (Rafal \& Inhoff, 1986) in which no cues were used to summon attention, we found no difference in detection RT between temporal and nasal hemifields.

For the single-cue trials, in which we measured the initial facilitatory effect of the cue, there was no advantage for targets appearing at the cued location in the temporal hemifield, in comparison with those in the nasal hemifield. This part of our results confirms those of Shulman, Wilson, and Sheehy (1985) and Rafal and Inhoff (1986), who also found no temporal hemifield advantage for covert orienting of attention, and it supports the argument of Shulman et al. (1985) and of Posner and Cohen (1980) that midbrain pathways are more important for overt orienting (i.e., eye movements) than for covert orienting of attention.

In contrast, Posner and Cohen (1984) suggested that inhibition of return was more intimately related with eye move- 
ments, and it was this proposal that led us to investigate the phenomenon in patients with eye-movement impairment from midbrain lesions (Posner et al., 1985) and to measure it in temporal and nasal hemifields in normal humans in this study. Experiment 1 showed that inhibition of return was larger in the temporal hemifield under monocular conditions. This result converges with our observations in patients with midbrain lesions and is consistent with the hypothesis that inhibition of return is mediated through activation of the retinotectal pathway.

In Experiment 1, as in previous studies (Maylor, 1985; Maylor \& Hockey, 1985; Posner \& Cohen, 1984; Tassinari et al., 1987), inhibition of return is inferred from the slower RTs to targets appearing at a previously cued location, in comparison with those appearing in the contralateral visual field. Is this RT difference, however, the result of inhibition at the cued location or of facilitation of responses to contralateral targets? According to Figure 1, there are, in general, quicker RTs for the later intervals. This pattern is expected because uncertainty for target appearance decreases at the later intervals. This RT advantage for later cue-target intervals is not manifest for targets appearing at the cued location in doublecue trials. Posner and Cohen (1984) observed this same pattern in their experiments and concluded that it was inhibition that truncated the expected effect of interval for this condition. Our interpretation of Experiment 1 is predicated on the assumptions that expectancy effects should produce faster RTs on double-cue trials than on single-cue trials and that the lack of faster RTs for targets at the first cued location on double-cue trials reflects an inhibitory process. Our interpretation is also predicated on the assumption that there is no inherent advantage of nasal field detection over temporal field. In Experiment 2 we tested the validity of these assumptions. In this experiment the display, instructions, and cuetarget intervals were identical to those of Experiment 1, except that the first cue was always brightening of the center box. We predicted that there would be no differences in RT for targets in temporal and nasal hemifields and that RTs would be faster in double-cue trials than in single-cue trials.

\section{Experiment 2: Detection of Targets in Temporal and Nasal Hemifields After Central Cues}

\section{Method}

Eight adult subjects were tested under monocular conditions, as in Experiment 1. In this experiment the first cue was brightening of the center box for $300 \mathrm{~ms}$. The intertrial interval was $1,000 \mathrm{~ms}$. In all other respects the display, the instructions, and the procedure were identical to those of Experiment 1.

\section{Results and Discussion}

Mean RTs for Experiment 2 are shown in Figure 3. RTs were faster for the double-cue trials, $F(3,24)=30.0, p<$ .001 . RTs did not differ between temporal and nasal hemifields, $F(1,8)=0.01$.

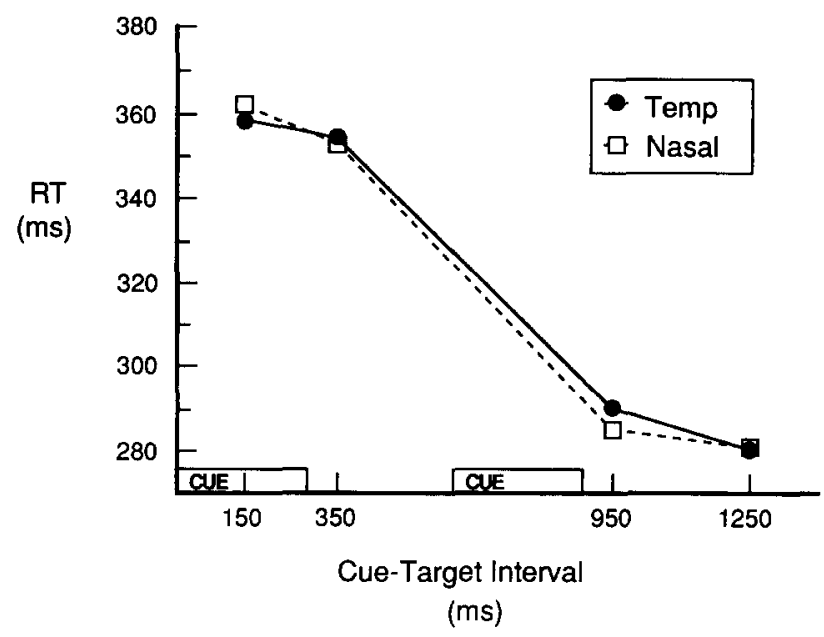

Figure 3. Mean reaction times (RTs) from Experiment 2. (RTs for targets appearing in temporal and nasal hemifields as a function of cue-target interval.)

The results of this control experiment are consistent with our interpretation of Experiment 1 that a signal presented in the peripheral visual field produces an inhibition of subsequent detection and that this inhibition of return is larger in the temporal hemifield. Nevertheless, we have not specifically addressed the issue of whether the inhibition of return (Posner $\&$ Cohen, 1984) is in fact a purely inhibitory phenomenon or whether there might also be a facilitation of responses to contralateral targets. We conducted Experiment 3 to examine this issue by incorporating a neutral cue (central-brightening first cue) condition that would permit the identification of either costs (i.e., inhibition) ipsilateral to peripheral cues or benefits (i.e., facilitation) in the field contralateral to peripheral cues.

\section{Experiment 3: Costs and Benefits Deriving From "Inhibition of Return"}

In Experiment 1 we used the method originally described by Posner and Cohen (1984) to generate inhibition of return; that is, a peripheral cue that predicted likely target location was first presented to summon attention and was followed by a center's brightening to bring attention back to the center. As shown again in Experiment 1, this paradigm is effective in producing response inhibition to targets at recently attended locations. Maylor (1985) and Tassinari et al. (1987) showed more recently, however, that a peripheral visual signal automatically produces a biphasic effect in responding to targets at the location of the signal. Even if the cue does not predict the likely target location, and even if no manipulation is used to summon attention back to the center of the display, a biphasic pattern is seen: For the first $200-300 \mathrm{~ms}$, detection RT is quicker to targets at the location of the peripheral signal; for longer cue-target intervals, detection RTs are slower at that location. 
In Experiment 3 we measured inhibition of return activated in this way in temporal and nasal hemifields. Our intent was to confirm that inhibition of return generated by this kind of reflexive orienting was reliably larger when activated by signals in the temporal hemifield. In addition, we incorporated a neutral-cue condition, in which the first cue could be brightening of the center box, so that we could identify a relative ipsilateral inhibition or contralateral facilitation, or both, produced by peripheral signals, in comparison with the center-brightening (neutral-cue) condition. Each trial began with brightening of one of the three boxes (left, center, or right) with equal probability. The first cue was never followed by a target, but always by a center brightening, which was then followed by the target, which appeared with equal probability in either of the two peripheral boxes. In this experiment, then, we did not measure the early summoning of attention by peripheral signals; rather, we focused exclusively on the subsequent inhibition of return.

\section{Method}

Subjects. Seventeen normal young adults were paid to participate. They were tested in a single session under monocular conditions, first with one eye patched, then the other.

Apparatus. The apparatus and display were the same as that used for Experiment 1.

Procedure. After an intertrial interval of $1,000 \mathrm{~ms}$, each trial began with the offset of the central + sign, followed $500 \mathrm{~ms}$ later by the appearance of the first cue. The first cue was the brightening, with equal probability, of one of the three boxes (left, right, or center) for $300 \mathrm{~ms}$. Five hundred milliseconds after onset of the first cue, the center box brightened for $300 \mathrm{~ms}$. The target then appeared, with equal probability, in either of the two peripheral boxes at an interval of $950,1,100,1,250$ or $1,400 \mathrm{~ms}$ after onset of the first cue. The target remained visible until subjects responded. As in Experiment 1, subjects were instructed to maintain fixation on the center of the display and to make a simple key-press response whenever the target appeared in either of the two peripheral boxes.

\section{Results}

After exclusion of responses of less than $100 \mathrm{~ms}$ (less than $2 \%$ ), median RTs from each subject for each condition were analyzed in a $2 \times 3 \times 4$ repeated-measures ANOVA. Withinsubject factors included field (temporal or nasal), cue (ipsilateral to peripheral cue, contralateral to peripheral cue, or central cue), and interval $(950,1,100,1,250$ or $1,400 \mathrm{~ms})$.

RTs were systematically faster for the later cue-target intervals, $F(3,48)=61.1, p<.001$; however, interval did not interact with any of the other factors. Mean RTs for the six conditions in Experiment 3 are shown in Table 1.

RTs were slower for targets appearing ipsilateral to peripheral cues ( $357 \mathrm{~ms}$ ) than in the neutral-cue condition (340 ms) and were faster for targets appearing contralateral to peripheral cues $(327 \mathrm{~ms})$ than in the neutral condition, $F(2,32)=$ $20.4, p<.001$. There was a main effect of hemifield: RTs to nasal targets were faster, $F(1,16)=6.1, p<.025$. The effect of cue was larger in the temporal than in the nasal hemifield, $F(2,32)=4.2, p<.025$.
Table 1

Mean Reaction Times (in ms) for Experiment 3

\begin{tabular}{ccc}
\hline Condition & $\begin{array}{c}\text { Temporal } \\
\text { hemifield }\end{array}$ & $\begin{array}{c}\text { Nasal } \\
\text { hemifield }\end{array}$ \\
\hline Cued location & & \\
$M$ & 363 & 351 \\
$S D$ & 55 & 44 \\
Neutral cue & & \\
$M$ & 343 & 335 \\
$S D$ & 49 & 41 \\
Uncued location & & \\
$M$ & 326 & 328 \\
$S D$ & 49 & 50 \\
\hline
\end{tabular}

Note. "Cued location" means that the target appeared at the location indicated by the first cue. "Uncued location" means that the target appeared in the field contralateral to the cued location. In the "neutral cue" condition, the first cue was the brightening of the center box, and neither of the possible target locations had been cued.

Post hoc comparisons of data from the peripheral cue conditions revealed that for targets appearing ipsilateral to the first cue, RTs were significantly slower for temporal than for nasal targets, $F(1,16)=7.0, p<.025$, whereas for targets appearing contralateral to the first cue, RTs in temporal and nasal hemifields did not differ, $F(1,16)=0.49$. For the neutral-cue condition, RTs were faster for nasal than for temporal targets, $F(1,16)=5.8, p<.05$.

\section{Discussion}

The results of Experiment 3 confirm the findings of Maylor (1985) and Tassinari et al. (1987) that a peripheral signal automatically generates an inhibition of return even under circumstances in which it does not predict the likely location of any target. In this sense, inhibition of return appears to be reflexive. Furthermore, these results confirm those of Experiment 1 that signals presented to the temporal hemifield generate a more robust inhibition of return effect. Last, the results of Experiment 3 confirm that the slower responses to targets at the location of the peripheral signal result from an inhibitory process. Under the conditions of this experiment, detection of targets contralateral to a peripheral cue were facilitated, in comparison with the neutral (center-brightening) first-cue condition. This aspect of our results should not be interpreted to mean that inhibition of return is always accompanied by a specific facilitation of responses to targets at the homologous location in the contralateral hemifield. In Experiment 3, the target always occurred at one of the two locations. Therefore, a bias against responding to signals at one of the two locations should be expected to be associated with a bias to respond to targets at the other. Under circumstances in which several target locations are possible, the apparent facilitation that we found in Experiment 3 need not necessarily occur. In one experiment (Posner \& Cohen, 1984) the target could occur not only contralateral to a peripheral cue but also above or below fixation. Thus the target could occur either at the cued location or at one of three other locations. In that experiment, detection RTs were slower at 
the cued location than at any of the other three locations, and RTs were not different among the other three.

One unexpected result of this experiment was that in the neutral-cue condition, RTs were faster for targets in the nasal hemifield than in the temporal hemifield. Because we have shown that there is no systematic difference for target detection between temporal and nasal hemifields in no-cue (Rafal \& Inhoff, 1986) or neutral-cue (Experiment 2) experiments in which there are no peripheral cues, we do not have a satisfactory explanation for this finding. It may be that under certain circumstances, subjects may be slower to disengage attention from the point of fixation to move in a temporal than in a nasal direction. We believe another mechanism to be more likely. Inasmuch as the inhibition of return can be sustained for several seconds, it is quite possible, given the short (1,000-ms) ITI in the current experiment, that the effect generated in peripheral cue trials may spill over into neutral cue trials.

The greater inhibition of return in the temporal hemifield, shown in Experiments 1 and 3, does not, however, simply result from a slower temporal movement from the centerbrightening second cue. The same results are found even under circumstances in which there are no signals at the center. After completing Experiment 1, we (Rafal \& Inhoff, 1986) also measured inhibition of return in temporal and nasal hemifields, using the method described by Maylor (1985). Using the same apparatus and display employed in Experiment 1, we presented a single cue (brightening of a peripheral box for $200 \mathrm{~ms}$ ), which was followed by the target that appeared, with equal probability, in one of the two boxes. The cue-target intervals were $50,150,300$, and $500 \mathrm{~ms}$. The peripheral cue did not predict the location of any target, and no center brightening occurred at any time. The results were that RTs at the cued location were the same in the temporal and nasal hemifields for the 50-, 150-, and 300-ms intervals. At the 500-ms interval, however, detection RTs were slower at the cued location in the temporal hemifield than in the nasal hemifield.

The greater efficacy of signals in the temporal hemifield in activating inhibition of return appears, then, to be a reliable phenomenon that we have replicated in several different experimental situations. This asymmetry between temporal and nasal hemifields is reliably present, regardless of whether the peripheral cue predicts the location of any target, and even when no center brightening is used to summon attention back to the fixation point. We suggest that this temporal hemifield advantage reflects the neuroanatomic connections of the midbrain retinotectal pathway. These hemifield asymmetries in normal humans converge with our previous observations that neurological patients with peritectal degeneration show deficiency in the inhibition of return (Posner et al., 1985). These converging observations in normal humans and neurological patients are consistent with a midbrain mechanism.

In our conclusions we do not assume that the colliculus is entirely "blind" to the nasal hemifield. In adults the superior colliculus does receive binocular input, inasmuch as each colliculus receives afferents from the binocularly innervated striate cortex. Thus, the asymmetry of inhibition of return reported here could only reflect the predominance of direct connections from the temporal visual field to the colliculus. In fact, our experiment showed that inhibition of return was present in both hemifields. We have interpreted the relatively greater effect in the temporal hemifield to reflect an advantage conferred by its more direct connections to the colliculus.

This conclusion is predicated on the assumption that the asymmetric anatomic projections of nasal and temporal hemiretinas, shown in cats and monkeys, are relevant to human neuroanatomy and that such an arrangement would be functionally relevant in human adults with a fully developed geniculostriate pathway. Lewis et al. (1979) showed that newborn infants execute saccades chiefly toward targets in the temporal hemifield. They interpreted these results as reflecting a functional competence of the retinotectal pathway in newborns who do not yet have a functioning geniculostriate pathway.

Posner and Cohen (1980) showed that even in human adults, there is a persisting bias to make saccades toward the temporal hemifield. Under monocular conditions, their subjects were presented bilateral simultaneous targets and were instructed to make an eye movement toward "whichever felt most comfortable and natural." They found a strong bias to make saccades toward the temporal hemifield. These results suggest that a peripheral visual signal primes or biases the oculomotor system to prepare a saccade toward it and that this priming is mediated by the retinotectal pathway. In contrast, Posner and Cohen found no temporal hemifield advantage for perceptual (temporal order) judgments, which again suggests that visual attention, if not linked with eye movements, is less dependent on the retinotectal pathway.

We have shown that inhibition of return is more robust in the temporal hemifield. Also, like the oculomotor temporal bias shown by Posner and Cohen (1980), inhibition of return seems to have strong effects on biasing motor responses, but it does not seem to affect perceptual (temporal order) judgments (Posner et al., 1985). In our demonstrating the temporal hemifield advantage for inhibition of return, our main purpose was not to definitively localize its precise neuroanatomic substrate; rather, we wished to demonstrate that it seems to share a common pathway with oculomotor mechanisms, inasmuch as the hypothesis underlying this investigation is that inhibition of return is chiefly concerned with eye movement control. In Experiment 4 we sought to specify more precisely the relation between inhibition of return and eye movements.

\section{Experiment 4: Exogenous and Endogenous Activation of Inhibition of Return}

Posner and Cohen (1984) found that inhibition of return followed attention shifts summoned by a peripheral luminance change (either brightening or dimming), but not shifts of attention activated endogenously (from a symbol-an arrow-presented at the center of the display). They initially suggested that it was the result of sensory processes, rather than being related to attention. However, Posner et al. (1985) subsequently showed that inhibition of return could be gen- 
erated by endogenously activated saccades, even in the absence of a peripheral signal. In that experiment, subjects were presented an arrow in the center of the display that directed them to move their eyes to one side or the other. Subjects then had to read a small digit (which had been plotted in the box before the start of the trial) and then execute a saccade back to the center. After the eyes had returned to the center of the display, the target appeared with equal probability either in the box to which the saccade had just been made or in the contralateral box, and subjects made a manual keypress response to this target. Detection RT was slower at the location to which the saccade had been made.

Thus inhibition of return may be activated by an exogenous sensory event with the eyes fixed or by an endogenously generated eye movement. As discussed earlier, however, Posner and Cohen (1980) showed that an exogenous visual signal not only summons attention but also primes the oculomotor system to prepare a saccade through the retinotectal pathway. With regard to inhibition of return, we may then consider whether it could be activated as a result of this priming of the oculomotor system by exogenous signals, rather than as a direct result either of sensory processes (as suggested initially by Posner \& Cohen, 1984) or of orienting covert attention (as suggested by Maylor, 1985).

In Experiment 4 we explore the possibility that there are not two separate pathways for exogenous and endogenous activation of the inhibition of return but rather a common mechanism related to oculomotor preparation. If a signal in the visual periphery induces the preparation of an eye movement, as shown by Posner and Cohen (1980), and if it is this oculomotor priming that generates the inhibition of return, then it should be possible to activate the inhibition of return endogenously by preparing an eye movement, even under circumstances in which there is no peripheral signal and in which no eye movement is actually made. The fourth experiment was designed to test the hypothesis that priming of the oculomotor system to prepare a saccade is necessary and sufficient to produce inhibition of return.

\section{Method}

Subjects. Nineteen normal adults participated in this experiment. Apparatus. The apparatus and display were the same as for Experiment 1.

Procedure. Subjects were tested under binocular conditions in six separate blocks of 160 trials each. The six experimental conditions constituted an orthogonal design that varied along two dimensions: (a) type of cue (exogenous cuing or endogenous cuing) and (b) eye movement instruction (eyes fixed, executing saccade, and preparing saccade).

In the exogenous cuing condition, the cue was the brightening of a peripheral box, as in Experiment 1 (see Figure 1). In the endogenous cuing condition, the display and the cue and target probabilities were the same as the exogenous cuing condition except that the cue was the presentation, at the center of the display, of an arrow, $1^{\circ}$ of visual angle across, pointing either left or right. Both types of cues were, of course, exogenous visual signals. The terms exogenous and endogenous cuing here refer to the means by which attention shifts and eye movements were instructed. Because with the arrow cue a target never appeared at the physical location of the cue, any effects that it produced at the locations of subsequent targets could have been generated only endogenously, on the basis of its symbolic meaning.

The three eye movement instruction conditions are summarized in Table 2. In the eyes-fixed condition, subjects were required to keep their eyes fixed on the center box constantly throughout the task and to respond to targets (on either single-cue or double-cue trials) with a quick key press. In the saccade-execution condition, subjects were instructed to make a quick eye movement toward the cued box immediately on the appearance of the first cue and then to press the response key quickly if the target appeared after this cue. However, on the double-cue trials, subjects were instructed to move their eyes quickly back to the center box when it brightened and then to press the response key quickly when the target subsequently appeared. Thus for the double-cue (center-brightening) trials in this condition, targets were preceded by a saccadic eye movement to one box and the return of the eyes to the center before the target appeared. In the saccade-preparation condition, subjects were instructed to "get ready" to move their eyes to the cued box after the first cue but to execute the saccade only if and when the target actually appeared. For the single-cue trials, subjects were required to make a saccade to the target wherever it occurred. However, on the double-cue (centerbrightening) trials, subjects were instructed to "cancel" the eye movement if the center box brightened, to maintain fixation, and to press the response key quickly when the target appeared. Thus in this condition, on these double-cue trials in which there was a central brightening, the subjects had prepared a saccade in one direction but had not made it at the time that the target eventually appeared.

In this experiment the intertrial interval was $3,000 \mathrm{~ms}$. The cue remained visible for $500 \mathrm{~ms}$ and, in half of the trials (single-cue trials), the target appeared either 450 or $650 \mathrm{~ms}$ after the onset of the first cue. The target appeared, in these trials, at the cued location $80 \%$ of the time. In the other half of the trials (double-cue trials), no target appeared after the first cue; instead, the center box brightened 700 $\mathrm{ms}$ after the onset of the first cue and remained visible for $300 \mathrm{~ms}$. On these center-brightening trials, the target appeared with equal probability in either peripheral box, 950 or $1,250 \mathrm{~ms}$ after the onset of the first cue. There were no catch trials.

Each subject was tested separately for each of the three eye movement conditions on three different days. For each eye-movement instruction condition, both the arrow cue blocks and the peripheral box-brightening cue blocks were tested in the same session; the order was randomized across subjects. All subjects were tested in the eyesfixed conditions on the first testing day in order to become familiarized with the task. The order of the other two eye-movement instruction conditions was then randomized across subjects. The same order of cue type was maintained for each subject for each of the three eyemovement instruction conditions. For each of the four blocks in which the subjects had to make or inhibit eye movements, they were given a practice block of 40 trials. After practice, most subjects executed all trials without making any eye-movement errors; for no subject did the number of eye-movement errors exceed $3 \%$ of the critical center-brightening trials.

During both the practice and the experimental blocks, the experimenter closely monitored eye position with a video camera to ensure compliance with the instructions. A dim red light was used to produce a light reflex off the cornea, which allowed the experimenter to detect any eye movement of $3^{\circ}$ or more. Our method of recording eye movements did not allow us to measure saccade latency or accuracy.

\section{Results}

For the single-cue trials, RT data were available only from the eyes-fixed and the saccade-execution blocks (because no key-press response was made in the saccade-preparation 
Table 2

Instructions to Subjects for Experiment 4

\begin{tabular}{|c|c|c|c|c|}
\hline \multirow[b]{2}{*}{ Condition } & \multicolumn{2}{|c|}{ Single cue } & \multicolumn{2}{|c|}{ Double cue } \\
\hline & Cue on & Target on & Center on & Target on \\
\hline Eyes fixed & Maintain fixation & Press key & Maintain fixation & Press key \\
\hline Saccade execution & Saccade to cue & Press key & $\begin{array}{l}\text { Saccade back to } \\
\text { center }\end{array}$ & Press key \\
\hline Saccade preparation & $\begin{array}{l}\text { Prepare saccade } \\
\text { to cue }\end{array}$ & $\begin{array}{l}\text { Saccade to } \\
\text { target }\end{array}$ & $\begin{array}{l}\text { Cancel prepared } \\
\text { saccade }\end{array}$ & Press key \\
\hline
\end{tabular}

blocks on single-cue trials.) For this reason, the data from single-cue and double-cue trials were analyzed separately.

Single-cue trials. Anticipatory responses (RT $<100 \mathrm{~ms}$ ) occurred in less than $2 \%$ of trials and were excluded from subsequent analysis. The mean data from the single-cue trials were then analyzed in a $2 \times 2 \times 2 \times 2$ ANOVA. Within-subject factors were instruction (eyes-fixed or saccade-execution), cue type (box or arrow), cue validity (target appearing at cued location or target contralateral to cued location), and interval (450 or $650 \mathrm{~ms}$ ). The mean RTs are shown in Table 3.

Manual RTs were slower in the saccade-execution conditions than in the eyes-fixed conditions, $F(1,18)=117.4, p<$ .001 . In the saccade-execution conditions, RTs were quicker for box than for arrow cues, resulting in an Instruction $\times$ Cue Type interaction, $F(1,18)=11.5, p<.005$. In the saccadeexecution conditions, RTs were slower at the 450 -ms than at the 650 -ms cue-target interval, resulting in an Instruction $x$ Interval interaction, $F(2,18)=4.9, p<.05$. RTs were quicker to targets appearing at the cued location than to targets appearing contralateral to it in all four conditions, $F(1,18)=$ $57.2, p<.001$. This effect of cue validity was greater for arrow than for box cues, $F(1,18)=16.1, p=.001$. For both conditions with a box cue, the effect of cue validity was greater

Table 3

Mean Reaction Times (in ms) for Single-Cue Trials in Experiment 4

\begin{tabular}{|c|c|c|c|c|}
\hline \multirow[b]{3}{*}{ Target position } & \multicolumn{4}{|c|}{ Cue-target interval (in ms) } \\
\hline & \multicolumn{2}{|c|}{ Box cue } & \multicolumn{2}{|c|}{ Arrow cue } \\
\hline & 450 & 650 & 450 & 650 \\
\hline & \multicolumn{2}{|c|}{ Eyes fixed } & & \\
\hline \multicolumn{5}{|l|}{ Cued location } \\
\hline$M$ & 363 & 367 & 337 & 341 \\
\hline$S D$ & 46 & 51 & 56 & 62 \\
\hline \multicolumn{5}{|l|}{ Uncued location } \\
\hline$M$ & 371 & 388 & 371 & 376 \\
\hline$S D$ & 50 & 51 & 58 & 81 \\
\hline \multicolumn{5}{|c|}{ Saccade execution } \\
\hline \multicolumn{5}{|l|}{ Cued location } \\
\hline$M$ & 502 & 482 & 554 & 535 \\
\hline$S D$ & 68 & 51 & 123 & 78 \\
\hline \multicolumn{5}{|l|}{ Uncued location } \\
\hline$M$ & 581 & 584 & 675 & 624 \\
\hline$S D$ & 117 & 74 & 160 & 116 \\
\hline
\end{tabular}

Note. "Cued location" means that the target appeared at the location indicated by the cue. "Uncued location" means that the target appeared in the field contralateral to the cued location. at the 650 -ms than at the 450 -ms cue-target interval, resulting in a Cue Type $\times$ Cue Validity $\times$ Interval interaction, $F(1,18)$ $=7.5 . p<.025$. However, the effect of cue validity for the two cue-target intervals did not differ between the eyes-fixed and the saccade-execution conditions.

Double-cue trials. RTs of less than $100 \mathrm{~ms}$ or greater than 3,500 ms were excluded from further analysis; this led to the exclusion of less than $4 \%$ of trials from the eyes-fixed condition and less than $2 \%$ of trials from the eye-movement conditions. We calculated mean RTs for each subject, for each of the four trial types that followed a center's brightening (i.e., cued and uncued target locations; $950-\mathrm{ms}$ and 1,250-ms cuetarget intervals), and the means for all 19 subjects were subjected to a $3 \times 2 \times 2 \times 2$ ANOVA incorporating all six conditions. Within-subject factors included instruction (eyes fixed, saccade execution, or saccade preparation), cue type (box or arrow), cue validity (target's appearing ipsilateral to cue or contralateral to cue), and interval (950 or $1,250 \mathrm{~ms}$ ). In addition, we conducted a separate ANOVA with the data in each of the six conditions. Mean RTs from the double-cue trials for each of the six conditions are shown in Table 4.

Manual RTs were quicker for the eyes-fixed condition than for the other four conditions in which saccades had to be executed or inhibited, $F(2,36)=34.7, p<.001$. RTs were quicker for the longer $(1,250$-ms $)$ cue-target interval, $F(1,18)$ $=182.9, p<.001$. This advantage for the longer interval was greater for the saccade-execution condition $F(2,36)=49.5$, $p<.001$, and was also somewhat greater for the arrow cue than for the box cue conditions, $F(1,18)=5.9, p<.025$. RTs were quicker with arrow cues in the saccade-preparation condition and with box cues in the saccade-execution condition, which resulted in an Instruction $\times$ Cue Type interaction, $F(2,36)=5.0, p<.025$. There was a main effect of cue validity: RTs were slower for targets appearing at the cued location than for those contralateral to the cued location, $F(1$, 18) $=21.9, p<.001$. This inhibition-of-return effect was present in each of the six conditions except for the eyes-fixed, arrow-cue condition; this resulted in a Cue Validity $\times$ Cue Type $\times$ Instruction interaction, $F(2,36)=4.1, p<.025$. Separate ANOVAs done on each of the six conditions confirmed that there was an inhibition-of-return effect in all but the eyes-fixed, arrow-cue condition (see Table 5).

A critical comparison in this experiment is between the eyes-fixed, arrow-cue condition and the saccade-preparation arrow-cue condition. In the center-brightening trials, the visual display and the overt behavior of the subjects were the same. In neither was there a peripheral cue, nor was any eye movement made. The only difference between these conditions was the instructions to subjects. The results indicated 
Table 4

Mean Reaction Times (in ms) for Double-Cue Trials in Experiment 4

\begin{tabular}{|c|c|c|c|c|}
\hline \multirow[b]{3}{*}{ Target positon } & \multicolumn{4}{|c|}{ Cue-target interval (in ms) } \\
\hline & \multicolumn{2}{|c|}{ Box cue } & \multicolumn{2}{|c|}{ Arrow cue } \\
\hline & 950 & 1250 & 950 & 1250 \\
\hline \multicolumn{5}{|c|}{ Eyes fixed } \\
\hline \multicolumn{5}{|l|}{ Cued location } \\
\hline & 361 & 357 & 319 & 294 \\
\hline$S D$ & 39 & 37 & 58 & 60 \\
\hline \multicolumn{5}{|l|}{ Uncued location } \\
\hline$M$ & 329 & 299 & 323 & 296 \\
\hline$S D$ & 45 & 43 & 59 & 73 \\
\hline \multicolumn{5}{|c|}{ Saccade execution } \\
\hline Cued location & & & & \\
\hline$M$ & 470 & 382 & 520 & 386 \\
\hline$S D$ & 107 & 110 & 110 & 100 \\
\hline \multicolumn{5}{|l|}{ Uncued location } \\
\hline$M$ & 444 & 338 & 494 & 362 \\
\hline$S D$ & 103 & 76 & 107 & 99 \\
\hline \multicolumn{5}{|c|}{ Saccade Preparation } \\
\hline Cued location & & & & \\
\hline$M$ & 466 & 380 & 446 & 344 \\
\hline$S D$ & 106 & 115 & 110 & 106 \\
\hline \multicolumn{5}{|l|}{ Uncued location } \\
\hline$M$ & 444 & 343 & 416 & 322 \\
\hline$S D$ & 104 & 75 & 124 & 108 \\
\hline
\end{tabular}

Note. "Cued location" means that the target appeared at the location indicated by the cue. "Uncued location" means that the target appeared in the field contralateral to the cued location.

that the instruction to prepare a saccade was effective in generating inhibition of return, whereas orienting covert attention without oculomotor preparation was not. An ANOVA in which we compared these two conditions confirmed the significance of this Instruction $\times$ Cue Validity interaction, $F(1,18)=6.1, p<.025$.

Given that both exogenous signals or oculomotor priming may be effective in generating inhibition of return, we may also consider whether these are independent effects that may make additive contributions to inhibition of return. Is oculomotor priming that is activated by exogenous signals more effective in generating inhibition of return than when oculomotor priming is generated endogenously? To address this question, we cast the four eye movement conditions (saccadeexecution, box cue; saccade-execution, arrow cue; saccadepreparation, box cue; and saccade-preparation, arrow cue) into a separate ANOVA. This analysis confirmed the Instruction $\times$ Cue Type interaction discussed earlier. There were, however, no interactions between cue validity and cue type, $F(1$, $18)=0.19$, or between cue validity and instruction, $F(1,18)$ $=0.07$.

\section{Discussion}

Before we focus on the results from the double-cue trials, in which inhibition of return was measured, several findings from the single-cue results deserve consideration. Reaction time was slower in the saccade-execution conditions than in the eyes-fixed condition. The saccade conditions were dual tasks; subjects had to make both eye movements and hand movements. Posner, Nissen, and Ogden (1978) previously noted that manual responses are slowed in tasks that also require eye movements. From this observation they concluded that execution of eye movements require attention and are not, therefore, entirely automatic. Within the saccadeexecution conditions, there was also an interaction with cue type; RTs were slower with arrow cues than with box cues. Posner, Crippin, Cohen, and Rafal (1986) made a similar observation and concluded that a visual signal at fixation (the arrow cue in this case) commands attention, which must be disengaged before a response (with either the hand or the eyes) can be made to an eccentric target. Moreover, in the saccade-execution conditions, RT was slower for the earlier (450-ms) cue-target interval. Because we were not able to monitor eye position or the relative timing of eye movement and target appearance, this result is more difficult to interpret. One possibility is that at the 450 -ms interval, the eyes were still moving, at least on some trials. Saccadic suppression on these trials could have delayed detection of the target and thereby prolonged RT.

The most important result from the single-cue trials is that RTs were quicker to targets appearing at the cued location for all four conditions in which manual RT was recorded. This effect of cue was, of course, greater in the saccadeexecution than in the eyes-fixed conditions: In the saccade condition, the target was at fixation for valid cue trials, and it was $20^{\circ}$ eccentric on invalid trials. Thus for the saccadeexecution conditions, the effect of cue reflected not just attention but eye position. For the eyes-fixed conditions, the effect of cue is an index of covert attention. Both the box and the arrow cues were effective in summoning attention. The arrow cue with the eyes fixed was at least as effective in summoning attention as the box cue was. This is important because, as we discuss later, it was only in this condition that no inhibition of return was found on the double-cue trials. Thus the failure of endogenous cuing in the eye-fixed, arrowcue condition to activate inhibition of return cannot be ascribed to any failure of the cue to summon attention. Also,

Table 5

Inhibition of Return From Double-Cue Trials for Each Condition in Experiment 4

\begin{tabular}{ccc}
\hline & \multicolumn{2}{c}{ Cue type } \\
\cline { 2 - 3 } Instruction & Box cue & Arrow cue \\
\hline Eyes fixed & & \\
$M$ & $45^{* * * * *}$ & -3 \\
$F(1,18)$ & 88.7 & 0.4 \\
Saccade execution & & \\
$M$ & $35^{* *}$ & $25^{* * *}$ \\
$F(1,18)$ & 7.0 & 10.1 \\
Saccade preparation & & \\
$M$ & $30^{*}$ & $26^{*}$ \\
$F(1,18)$ & 4.8 & 5.0 \\
\hline
\end{tabular}

Note. The inhibition of return effect, shown in ms for each of the six conditions, is mean RT for targets appearing at the location indicated by the first cue minus mean RT for targets appearing in the field contralateral to the cued location.

${ }^{*} p<.05 . \quad{ }^{* *} p<.025 . \quad{ }^{* * *} p=.005 . \quad{ }^{* * * *} p<.001$. 
there was no interaction among instruction, cue validity, and interval. Thus if saccadic suppression was affecting subjects' performance, it seems to have done so without affecting the shifting of attention.

In the double-cue conditions, RT was again quicker in the eyes-fixed conditions than in the eye-movement conditions. RTs were also quicker with arrow cues in the saccade-preparation condition and with box cues in the saccade-execution condition. This result is understandable if one considers that saccades to peripheral signals are more automatic or reflexive. It is easier to make saccades to a peripheral signal than to generate them endogenously; conversely, it is harder to inhibit saccades to a peripheral signal than it is to inhibit endogenously prepared saccades. The more difficult oculomotor instructions are accompanied by longer manual responses.

The purpose of this experiment was to determine the necessary and sufficient conditions for activating inhibition of return as measured by the effect of cue validity in the doublecue trials. Inhibition of return occurred in all conditions except the endogenous cuing (arrow), eyes-fixed condition. We interpret this result to suggest that inhibition of return is activated through the oculomotor system, rather than resulting directly either from sensory processes or from orienting covert attention.

Posner and Cohen's (1984) finding that inhibition of return was activated by exogenous signals, but not by endogenously generated attention movements (from a central arrow cue), led them to speculate that it was a consequence of sensory rather than attention processes. Maylor (1983) showed, however, that it cannot be due to simple retinal adaptation or masking because the phenomenon exhibits complete interocular transfer, and it persists for a longer duration than these mechanisms would permit. It is not restricted to the specific site of sensory stimulation; rather, it spreads to nearby locations (Maylor \& Hockey, 1985) and, under certain circumstances, to the entire hemifield of the signal (Tassinari et al., 1987).

Maylor (1985) showed that a peripheral visual signal was not sufficient to produce inhibition of return because it could be prevented by circumstances that interfered with orienting attention to the signal (by giving subjects a competing oculomotor task). She argued that "externally triggered" attention movements were necessary to activate inhibition of return. However, Posner et al. (1985) showed that a peripheral signal was not always necessary to produce inhibition of return; one could also produce the phenomenon by executing a saccade, even when the saccade was made in response to a central arrow cue with no peripheral visual signal.

Thus inhibition of return is activated when attention is summoned by an exogenous signal or when attention is deployed endogenously in concert with eye movements. Experiment 4 was designed to test the hypothesis that there are, not two separate mechanisms for activating inhibition of return exogenously and endogenously, but a common mechanism underlying both. Posner and Cohen (1980), in demonstrating a temporal hemifield saccadic bias under conditions of bilateral stimulation, provided evidence that exogenous signals not only summon attention but also activate the oculomotor system. We speculated that it might be this oculomotor activation produced by exogenous signals, rather than sensory or attention processes, that was responsible for activation of inhibition of return. This speculation was prompted by several similarities between Posner and Cohen's (1980) temporal saccadic bias result and the inhibition of return. Experiments 1 and 3 showed that inhibition of return exhibited a temporal hemifield bias, suggesting that both may be mediated by retinotectal pathways. Both effects relate to the biasing of motor responses, rather than influencing perceptual processing. For example, Posner and Cohen (1980) showed that there was no temporal-nasal hemifield asymmetry in making temporal order judgments. Similarly, although inhibition of return affects the latency of simple and choice manual RT (Maylor, 1985), the latency of saccades (Vaughan, 1984), and the selection of saccade direction (Posner et al., 1985), it has no effect on temporal order judgments (Posner et al., 1985).

Our hypothesis predicted that inhibition of return would result from oculomotor activation, regardless of whether it was produced by exogenous signals or endogenously. The results of Experiment 4 confirmed this prediction and demonstrated that neither an exogenous signal nor saccade execution was necessary to produce the effect. Inhibition of return did occur under all conditions in which the cue was a peripheral signal and that in which saccades were executed in response to endogenous cuing. However, the important result was that saccade preparation, in response to endogenous cuing, was as effective in activating the inhibition of return as was the actual making of an eye movement or the occurrence of an exogenous sensory signal. Furthermore, the occurrence of an exogenous signal did not make an additive contribution to the inhibition of return under saccade-preparation conditions. These results indicate that saccade preparation is necessary and sufficient to produce inhibition of return and suggest that it provides a common mechanism for both its exogenous and endogenous activation.

In the interpretation of these results, the critical comparison is between the arrow-cue, eyes-fixed condition and the arrowcue, saccade-preparation condition. Although the overt behavior of the subjects was the same in these two conditions (no eye movements were made in either condition), the former did not activate inhibition of return (even though it did produce an orienting of attention, as shown by the results of the single-cue trials in Table 3 ), whereas the latter did. The only difference between these two conditions was the instruction to first prepare ("get ready") and then to inhibit ("cancel") a saccade. These instructions were effective in inducing inhibition of return, but was it the "prepare" or the "cancel" saccade requirement that was the critical factor in producing inhibition of return?

Recently, Tassinari et al. (1987) suggested that peripheral visual signals presented to fixating subjects produce inhibition of return (which they called inhibitory aftereffect) because the subjects must suppress a natural reaction to look at the stimulus. They suggested that subjects "have to generate a central command that counteracts the natural orienting reaction and vetoes the eye movement" and that "this suppressive action is bound to have consequences for more general motor adjustments" (p. 67). We incorporated a test for this hypothesis into this experiment by including conditions under which saccades were made, as well as those in which saccades 
had to be inhibited. Insofar as the actual execution of a saccade produced inhibition of return and did so to the same degree when the saccade was summoned by a peripheral signal, it is clear that inhibition of eye movements is not necessary to produce the effect.

We agree, then, with Tassinari et al. (1987) that peripheral visual signals engender inhibition of return because they activate the oculomotor system and that its function is to inhibit subsequent motor responses. We find, however, that oculomotor activation is sufficient to produce the effect and that there is no requirement for subjects to inhibit saccades in order for it to occur. When the oculomotor system is activated, either by exogenous signals or by endogenous commands, and whether an eye movement is made or not, inhibition of return occurs.

This interpretation derives from the results of the critical arrow-cue, saccade-preparation condition. We must consider, however, whether oculomotor preparation is specific in activating inhibition of return or whether it may result from any selective motor preparation for an expected signal. In Experiment 5, therefore, we repeated the critical arrow-cue, saccadepreparation condition of Experiment 4, except that subjects were required to prepare one of two possible manual responses when they saw the cue. If our hypothesis is correct, and if oculomotor preparation is specific in activating inhibition of return, then preparing a specific manual response to a signal should not activate inhibition of return.

\section{Experiment 5: Preparation of Manual Movements}

\section{Method}

Eight normal young adults volunteered to participate and were tested under binocular conditions. The visual display was identical to that used for the arrow-cue conditions of Experiment 4. The instructions were similar to the saccade-preparation condition of Experiment 4 except that subjects were instructed to prepare, on seeing the first cue, a RT key-press response with the left hand. As in the previous experiments, the index finger of the right hand rested on a single response key. The index and middle fingers of the left hand rested on two other adjacent response keys. When the central arrow cue appeared, subjects were instructed to "get ready" to press with the left middle finger if it pointed to the left or with the index finger if the arrow pointed to the right. On the single-cue trials, subjects were required to make a manual $R T$ response by pressing the left middle finger for targets appearing on the left or pressing the left index finger for targets appearing on the right. On the double-cue trials, subjects were required, when the center box brightened, to "cancel" the prepared movement with the left hand and to make a simple RT keypress response with the right hand, regardless of which side the target occurred. On these center-brightening trials, then, subjects had prepared a specific motor response to the first cue but had made no overt response when the center box brightened. The requirements of this experiment were, therefore, identical to the arrow-cue, saccadepreparation condition of Experiment 4 except that a manual, rather than an oculomotor, response had been prepared.

\section{Results}

Errors. Anticipatory errors (RT $<100 \mathrm{~ms}$ ) accounted for less than $4 \%$ of the data and were excluded from subsequent analysis. Errors in which the wrong key was pressed were very rare for the double-cue trials (in which a simple $\mathrm{RT}$ response with the right index finger was required). For the single-cue trials, the most common errors were due to stimulus-response compatibility confusions, which led to responses with the wrong hand-that is, responding to the correct target but with the wrong hand (e.g., pressing the right index finger when a target appeared on the right for a single-cue trial). These resulted in error rates of less than $2 \%$ for left targets and of $7 \%$ for right targets. These errors were not the result of responding to the wrong target or of executing an inappropriately prepared motor response. Of the single-cue trials, $5 \%$ consisted of errors in which a motor response prepared in response to an invalid cue was executed (i.e., pressing the left index finger for a right target or the left middle finger for a left target).

Reaction time. Mean RTs for correct responses for each subject in each condition of cue and interval were calculated. For the single-cue trials, mean RTs for all 8 subjects were 462 $\mathrm{ms}$ for targets appearing at cued locations and $618 \mathrm{~ms}$ for targets appearing at uncued locations. This effect of cue (156 $\mathrm{ms}$ ) is substantially larger than that found in the previous simple RT experiments and suggests that subjects did process the cue and prepare motor responses based on it. For the double-cue (center-brightening) trials, mean RT for targets at cued locations ( $341 \mathrm{~ms}$ ) was not significantly different from mean RT for targets at uncued locations (333 ms), $F(1,15)$ $=1.0$; that is, there was not a significant inhibition of return in this experiment.

\section{Discussion}

In this experiment then, the preparation of a specific manual response in anticipation of a visual target expected at an eccentric location did not produce an inhibition of return. This result is consistent with our hypothesis that oculomotor activation is necessary and sufficient for generating inhibition of return. They do not, of course, exclude the possibility that other nonocular forms of motor preparation (e.g., reaching) might not generate inhibition of return. They do, however, indicate that inhibition of return is not a general consequence of any form of motor activity directed or planned in response to expected eccentric visual signals.

\section{Experiment 6: Hypometric Saccades}

Our argument that saccade preparation is necessary and sufficient to activate inhibition of return hinges on the assumption that in the critical arrow-cue, saccade-preparation condition of Experiment 4, our subjects did not make saccades. Because their heads were not restrained, and because our video monitoring did not permit us to reliably detect saccades of less than $3^{\circ}$, it is possible that some subjects, on some trials, did make small saccades that we did not detect. We therefore ran a control experiment to determine whether hypometric saccades could have been responsible for the inhibition of return found in this critical condition.

\section{Method}

The same 8 subjects tested in Experiment 5 participated in this experiment. The display and procedure were the same as in the arrow- 
cue, saccade-execution condition of Experiment 4, except for the following modifications: Small $(0.5 \%)$ squares of white tape were placed, one on each side of the screen, halfway between the center and the peripheral box (i.e., $5^{\circ}$ eccentric to fixation). Subjects were required, when the central arrow cue appeared, to execute a saccade to the small square on the side indicated by the cue. If the target then appeared (single-cue trials), they pressed the response key. On the double-cue trials they were required, when the center box brightened, to move their eyes back to the center and then respond with a key press when the target appeared. As in Experiment 4, the target always appeared in one of the peripheral $\left(10^{\circ}\right.$ eccentric) boxes and never at the location to which the saccade had been made. Thus on the centerbrightening (double-cue) trials, subjects had made a hypometric saccade in the direction designated by the arrow cue and had returned their eyes to the center before the target appeared. We were easily able to observe these $5^{\circ}$ saccades and to confirm that subjects were following the instructions.

\section{Results and Discussion}

On the single-cue trials, RTs were quicker on the cued side (483 $\mathrm{ms}$ ) than on the uncued side $(562 \mathrm{~ms})$. On the doublecue trials, there was no significant difference between RTs on the cued side $(332 \mathrm{~ms})$ and the uncued side $(329 \mathrm{~ms}), F(1$, 15) $=0.18$.

When a peripheral signal summons attention, inhibition of return not only occurs at the locus of the signal but may spread to adjacent locations. Although both Maylor and Hockey (1985) and Tassinari et al. (1987) found this spread for inhibition of return, their results differed quantitatively. Maylor and Hockey presented eccentric cues on the horizontal meridian and measured inhibition of return for targets appearing perpendicular to the cue, above or below it. They found that inhibition of return fell off quite rapidly within a few degrees from the cue location. Tassinari et al., on the other hand, presented their targets orthogonal to the position of the cue. They found a fairly uniform inhibition of return for targets in the hemifield of the cue, which fell off abruptly at the midline meridian.

Experiment 6 did not reveal any inhibition of return at locations eccentric to positions to which saccades had been made. It was not our purpose here, however, to explore the relation between saccade metrics and the inhibition of return, nor did our method of monitoring eye movements permit us to do so. Rather, it was our intent to show that inhibition of return is not contingent upon the execution of eye movements. The purpose of Experiment 6, then, was only to control for the possibility that hypometric saccades might have accounted for inhibition of return in the saccade-preparation condition of Experiment 4. Therefore, we kept the conditions identical to Experiment 4 and simulated the possible circumstances under which unobserved saccades may have led us to misinterpret the results of that experiment. Because no inhibition of return occurred under these conditions in which there were clearly observed hypometric saccades, it is very unlikely that such an explanation could have accounted for the results of Experiment 3.

\section{General Discussion}

In everyday life, the orienting of attention to a point of interest is usually accompanied by overt movements of the eyes, the head, or the body. This is so regardless of whether attention is summoned exogenously by a sensory signal, as in turning toward a movement seen out of the corner of the eye, or deployed endogenously, as in deciding to look both ways before crossing the street. Contemporary research has, however, shown the existence of neural mechanisms for covertly orienting internal attention that can operate independently of those controlling oculomotor activity and other motor systems for overt orienting (Posner, 1980).

Interest now focuses on determining how neural systems mediating the orienting of attention are coordinated with those controlling eye movements. It is known that when a saccade is made, attention moves to the target of the forthcoming saccade before the saccade begins. This is so whether the eyes are summoned by a peripheral signal (Posner, 1980; Posner \& Cohen, 1980) or whether the saccade is triggered endogenously by, for example, central arrow cues (Shepherd, Findlay, \& Hockey, 1986). Conversely, covert attention can be allocated endogenously to a spatial location without activating the oculomotor system or influencing saccade latency (Klein, 1979; Shepherd et al., 1986). Exogenous visual signals, on the other hand, appear to have an automatic and biphasic effect on both covert attention and eye movements: First, attention is summoned to the location of the signal to facilitate its processing, and the oculomotor system is biased to saccade to that location; this initial facilitation is succeeded by an inhibition of return that inhibits subsequent responses to signals at the same location.

In this investigation we focused on this inhibition of return as a mechanism for integrating attention with eye movements and for arbitrating between exogenous and endogenous requirements for orienting in the visual field. We found further evidence that it may be mediated through the retinotectal pathway and that it is closely related to eye movements. Evidence for retinotectal mediation of inhibition of return derives from observations that it is impaired by midbrain lesions that impair saccade generation (Posner et al., 1985) and that it dominates in the temporal hemifield under monocular conditions (Experiments 1 and 3). Our hypothesis that the inhibition of return is activated by priming of the oculomotor system was supported by Experiment 4, which showed that endogenous saccade preparation was sufficient to activate inhibition of return and that exogenous sensory stimulation did not sui generis contribute additively to the effect.

The activation of inhibition of return seems to occur regardless of whether the oculomotor system is activated by an exogenous signal or by endogenously generated saccades. Once activated by the oculomotor system, inhibition of return then biases subsequent eye movements; but, in addition, it seems to bias covert attention systems as well insofar as it may also slow arbitrary, nonoculomotor responses (such as a key press in our investigation). In contrast, endogenous shifts of covert attention can be made without activating the oculomotor system (Shepherd et al., 1986) and do not generate inhibition of return (Posner \& Cohen, 1984; and Experiment 4.)

The observations reported here on inhibition of return are consistent with Posner and Cohen's (1984) initial suggestion that its function is to favor novelty in strategic visual scanning. We suggest, furthermore, that it may function to coordinate 
the oculomotor system's responses to exogenous and endogenous information. Because, in everyday life, exogenous sensory signals are constantly competing with endogenous control for access to the oculomotor apparatus, an inhibitory mechanism for arbitrating between them is required. Clearly, there are adaptive advantages to orienting automatically to new sensory signals occurring in the visual periphery. It is just as clearly necessary for humans to be able to control visual attention endogenously under voluntary guidance. Although automatic orienting of attention in response to new and salient events occurring in our visual periphery serves an important defensive and social function, its tight linkage with the generation of inhibition of return may permit us to search our environment strategically, under voluntary control, without continual distraction by repeated extraneous stimulation. Further studies of the inhibition of return under conditions of competing exogenous and endogenous demands may provide further insight into how these systems are coordinated to provide humans with a unity of perceptual experience that permits an efficient interaction with both the external and internal environment. ${ }^{1}$

\footnotetext{
${ }^{1}$ Klein (1988) has shown recently that inhibition of return occurs at the location of distractors in conjunction search, but not feature search tasks. This observation supports Posner and Cohen's (1984) proposal that inhibition of return does facilitate serial visual search by functioning as a location "tagging" mechanism (p. 430).

\section{References}

Bashinski, H. S., \& Bacharach, V. R. (1980). Enhancement of perceptual sensitivity as the result of selectively attending to spatial locations. Perception \& Psychophysics, 28, 241-248.

Hendrickson, A., Wilson, M. E., \& Toyne, M. J. (1970). The distribution of optic nerve fibers in Macaca mulatta. Brain Research, $23,425-427$.

Hubel, D. H., LeVay, S., \& Wiesel, T. N. (1975). Mode of termination of retinotectal fibers in macaque monkey: An autoradiographic study. Brain Research, 96, 25-40.

Klein, R. (1979). Does oculomotor readiness mediate cognitive control of visual attention? In J. B. Long \& A. D. Baddeley (Eds.), Attention and performance VIII. Hillsdale, NJ: Erlbaum.

Klein, R. (1988). Inhibitory tagging system facilitates visual search. Nature, 334, 430-431.

Lewis, T. L., Maurer, D., \& Milewski, A. (1979). The development of nasal detection in young infants. Investigative Ophthalmology and Visual Science, 18(Suppl.), 271.

Maylor, E. A. (1983). Components of orienting in visual space. Unpublished doctoral thesis, University of Durham, Durham.

Maylor, E. A. (1985). Facilitory and inhibitory components of orienting in visual space. In M. I. Posner \& O. S. M. Marin (Eds.), Attention and performance XI (chap. 9). Hillsdale, NJ: Erlbaum.
}

Maylor, E. A., \& Hockey, R. (1985). Inhibitory component of externally controlled covert orienting in visual space. Journal of Experimental Psychology: Human Perception and Performance, 11, 777787.

Pollack, J. G., \& Hickey, T. L. (1979). The distribution of retinocollicular axon terminals in rhesus monkey. Journal of Comparative Neurology, 185, 587-602.

Posner, M. I. (1980). Orienting of attention. Quarterly Journal of Experimental Psychology, 32, 3-25.

Posner, M. I., \& Cohen, Y. (1980). Attention and the control of movements. In G. E. Stelmach \& J. Requin (Eds.), Tutorials in motor behavior (pp. 243-258). Amsterdam: North Holland.

Posner, M. I., \& Cohen, Y. (1984). Components of visual orienting. In H. Bouma \& D. Bouwhuis (Eds.), Attention and performance $X$ (pp. 531-556). London: Erlbaum.

Posner, M. I., Cohen, Y., \& Rafal, R. (1982). Neural systems control of spatial orienting. Philisophical Transactions of the Royal Society of London, B298, 187-198.

Posner, M. I., Crippin, P. J., Cohen, A., \& Rafal, R. D. (1986, November). Speed of covert orienting of attention and express saccades 1. Paper presented at the Meeting of the Psychonomics Society, Boston.

Posner, M. I., Nissen, M. J., \& Ogden, W. (1978). Attended and unattended processing modes: the role of set for spatial location. In H. L. Pick \& I. J. Saltzman (Eds.), Modes of perceiving and processing information (chap. 7). Hillsdale, NJ: Erlbaum.

Posner, M. I., Rafal, R. D., Choate, L., \& Vaughan, J. (1985). Inhibition of return: Neural basis and function. Cognitive Neuropsychology, 2, 211-228.

Rafal, R. D., \& Inhoff, A. W. (1986). Midbrain mechanisms for orienting visual attention. In C. Clifton, Jr. (Ed.), Program of the Eighth Annual Conference of the Cognitive Science Society (pp. 260-271). London: Erlbaum.

Shepherd, M., Findlay, J. M., \& Hockey, R. J. (1986). The relationship between eye movements and spatial attention. Quarterly Journal of Experimental Psychology, 38A, 475-491.

Shulman, G. L., Wilson, J., \& Sheehy, J. B. (1985). Spatial determinants of the distribution of attention. Perception \& Psychophysics, $37,59-65$.

Tassinari, G., Aglioti, S., Chelazzi, L., Marzi, C. A., \& Berlucchi, G. (1987). Distribution in the visual field of the costs of voluntarily allocated attention and the inhibitory after-effects of covert orienting. Neuropsychologia, 25, 55-72.

Vaughan, J. (1984). Saccades directed at previously attended locations in space. In A. J. Gale \& C. W. Johnson (Eds.), Theoretical and applied aspects of eye movement research (p. 143). Amsterdam: North Holland.

Wilson, M. E., \& Toyne, M. G. (1970). Retino-tectal and corticotectal projections in Macaca mulatta. Brain Research, 24, 395406.

Received August 17, 1987

Revision received November 7, 1988

Accepted November 8, 1988 